\title{
Lifespan development of stimulus-response conflict cost: similarities and differences between maturation and senescence
}

\author{
Shu-Chen Li • Dorothea Hämmerer • Viktor Müller • \\ Bernhard Hommel · Ulman Lindenberger
}

Received: 20 March 2008 / Accepted: 17 July 2008 / Published online: 21 November 2008

(C) Springer-Verlag 2008

\begin{abstract}
Age gradient of the mechanism of stimulusresponse conflict cost was investigated in a populationbased representative sample of 291 individuals, covering the age range from 6 to 89 years. Stimulus-response conflict cost, indicated by the amount of additional processing time required when there is a conflict between stimulus and response options, follows a U-shaped function across the lifespan. Lifespan age gradient of conflict cost parallels closely those of processing fluctuation and fluid intelligence. Individuals at both ends of the lifespan displayed a greater amount of processing fluctuation and at the same time a larger amount of conflict cost and a lower level of fluid intelligence. After controlling for chronological age and baseline processing speed, conflict cost continues to correlate significantly with fluid intelligence in adulthood and old age and with processing fluctuation in old age. The relation between processing fluctuation and conflict cost in old age lends further support for the neuromodulation of neuronal noise theory of cognitive aging as well as for theories of dopaminergic modulation of conflict monitoring.
\end{abstract}

S.-C. Li ( $₫)$ · D. Hämmerer · V. Müller · U. Lindenberger

Center for Lifespan Psychology,

Max Planck Institute for Human Development,

Lentzeallee 94, 14195 Berlin, Germany

e-mail: shuchen@mpib-berlin.mpg.de

B. Hommel

Leiden University Institute of Psychology,

Leiden, The Netherlands

\section{Introduction}

Relative to research on working memory, attention, or inhibition, a concerted effort devoted toward understanding "conflict monitoring" as a neurocognitive concept and phenomenon in its own right is a recent endeavor that only emerged in the past decade. Early behavioral findings from the 1970s foreshadowed the notion that, the detection of "conflict" in the processes of cognitive operations (e.g., such as error detection) plays an important role in information processing (e.g., Higgins \& Angel, 1970; Rabbitt, 1966). Contemporary cognitive monitoring research over the past 10 years has established that monitoring mechanisms - in terms of outcome, processing or response conflict-are important adaptive signaling functions that could serve the purpose of online dynamical reconfigurations of the neurocognitive system in order for it to be sufficiently flexible and goal-oriented (cf. Ridderinkhof \& van den Wildenberg, 2005).

Mechanisms subserving conflict monitoring operate in a variety of related functions, such as outcome monitoring, error detection, or conflict resolution. Emerging evidence suggests that various regions of the prefrontal cortex (PFC) as well as the anterior cingulate cortex (ACC) are implicated in processes of outcome monitoring, error processing, reward-based learning, and conflict resolution (e.g., Cohen, Aston-Jones, \& Gilzenrat, 2004; Egner \& Hirsh, 2005; Ridderinkhof, Ullsperger, Crone, \& Nieuwenhuis, 2004; Ullsperger \& von Cramon, 2004; Yeung, Botvinick, \& Cohen, 2004). According to some accounts, activities in ACC send signals about detected conflicts (Botvinick \& Cohen, 2004) or error likelihoods (Brown \& Braver, 2005) to the PFC in order to generate stronger, compensatory PFC representations to support or direct task-relevant processing. In a related vein, PFC cognitive control mechanisms 
may bias processing to enhance target-relevant information, thus resolving conflicts. For instance, activations in dorsal lateral PFC have also been shown to display increased functional connectivity to perceptual regions (e.g., fusiform face area when face stimuli were used) under high conflict conditions (Egner \& Hirsh, 2005). Furthermore, empirical evidence from animal models (e.g., Schultz, 2002) and human studies (e.g., Berns, McClure, Pagnoni, Montague, 2001; Dolan et al., 1995; Schott et al., 2004; see also Marschner et al., 2005) as well as various neurocomputational models (e.g., Frank, Seeberger, \& O'Reilly, 2004; Holroyd \& Coles, 2002; Yeung et al., 2004) all point to the involvements of frontal and midbrain dopaminergic systems in modulating cognitive monitoring mechanisms (see also Montague, Hyman, \& Cohen, 2004, for review).

Whereas evidence of neural correlates of different aspects of conflict monitoring as well as theories of neuromodulation of conflict monitoring are gradually consolidating (e.g., Botvinick, Braver, Barch, Carter, \& Cohen, 2001; Brown \& Braver, 2005; Holroyd \& Coles, 2002; Yeung et al., 2004; see also Botvinick, Cohen, \& Carter, 2004; Montague et al., 2004, for reviews), research on the maturation (e.g., Davies, Segalowitz, \& Gavin, 2004; Ladouceur, Dahl, \& Carter, 2007; Rueda, Posner, Rothbart et al., 2004; Santesso, Segalowitz, \& Schmidt, 2006) and senescence (Fernandez-Duque \& Black, 2006; Mathewson, Dywan, \& Segalowitz, 2005) of conflict monitoring mechanisms has only started. The commonly used conflict monitoring tasks (e.g., the Stroop, Flanker, and Go/NoGo tasks) have been applied to address questions of child cognitive development or cognitive aging independently. Thus, so far a synopsis of age-related differences in conflict monitoring and their neural correlates across the lifespan needs to pieced together from different sets of evidence that have been separately collected at the two ends of the lifespan. Children's performance levels on the so-called "conflict tasks" (e.g., flanker and Go/NoGo tasks) are below the level of younger adults (e.g., Davies et al., 2004; Rueda et al., 2004; Williams, Ponesse, Schachar, Logan, \& Tannock, 1999). Similarly, older adults' performances also compare unfavorably to those of younger adults (e.g., Mathalon et al., 2003; Mathewson et al., 2005; West, 2004; West \& Moore, 2005). Initial evidence also suggests that children (Davies et al., 2004) and older adults (Nieuwenhuis et al., 2002) are less able in detecting errors than younger adults. However, there is a lack of direct comparisons of children's performance with that of older adults. Hence, thus far, little is known about the similarities and differences between the maturation and senescence of conflict monitoring.

As for neural correlates of conflict monitoring, recent findings from cognitive neuroscience of child development (e.g., Sowell, Thompson, Holmes, Jernigan, \& Toga, 2003) and aging (e.g., Raz et al., 2005) reveal that PFC and ACC mature late during child development and decline relatively early during aging, relative to other brain areas, such as the medial temporal structures. Specifically, the maturational gradients of PFC and ACC are very protracted, with continuing development until late adolescence (Sowell et al., 2003). At the neurofunctional level, the magnitude of event-related potentials associated with incorrect responses (i.e., error-related negativity) has been found to increase from childhood to adulthood (Davies et al., 2004) and to decrease during aging (Nieuwenhuis et al., 2002). At the neurochemical level, age-related changes in dopaminergic mechanisms during child development and aging that underlie various aspects of cognitive processing are also well established (for reviews, see Bäckman, Nyberg, Lindenberger, Li, \& Farde, 2006b; Diamond, 1996). At the same time, a related but distinct line of research also indicates that individual differences in within-person trialby-trial processing fluctuations may be attributable to development-, aging-, or pathology-related deficiencies in dopaminergic modulations (e.g., see Castellanos \& Tannock, 2002; Diamond, Briand, Fossella, \& Gehlbach, 2004; Li, Lindenberger, \& Sikström, 2001; see MacDonald, Nyberg, \& Bäckman, 2006; Winterer \& Weinberger, 2004, for reviews). With respect to processing fluctuations, children and older adults tend to show higher levels of withinperson trial-by-trial RT variability (e.g., Li et al., 2004; Williams, Hultsch, Strauss, Hunter, \& Tannock, 2005). Furthermore, older individuals who show greater shortterm trial-by-trial variability tend also to show greater extent of longitudinal declines in tasks assessing executive control (e.g., Lövdén, Li, Shing, \& Lindenberger, 2007; MacDonald, Hultsch, \& Dixon, 2003) and less efficient functional brain activation in regions (e.g., inferior parietal cortex) supporting episodic memory (MacDonald, Nyberg, Sandblom, Fischer, \& Bäckman, 2008).

Given that studies on the development and aging of conflict monitoring mechanisms have thus far been conducted separately, direct comparisons between the child developmental and aging gradients are lacking. To bridge the gap, we investigated lifespan development of conflict monitoring in a sample that covered the age range from 6 to 89 years. Specifically, we assessed conflict cost associated with the incongruence between stimulus and response to indicate individual differences in the efficacy of conflict monitoring mechanisms broadly defined. Furthermore, in order to understand the ontogenetic antecedents and consequences of age-related differences in conflict monitoring, at one level we examined the relations between the efficacy of conflicting monitoring and indicators of processing fluctuation, which could reflect the efficacy of dopaminergic modulation; and at another level, we examined the relation between conflict monitoring and fluid intelligence, which is supported by basic cognitive processes. 


\section{Method}

\section{Study sample}

The participants of this study were part of a populationbased representative sample of a large multi-session multivariate study on lifespan transformations in the functional organizations of intellectual abilities and their underlying basic cognitive and sensorimotor processes (the CoOPMIND Study, Drewing, Aschersleben, \& Li, 2006; Hommel, Li, \& Li, 2004; Li et al., 2004; Waszak, Schneider, Li, \& Hommel, in press). The sample was stratified by age and sex. After excluding participants who missed multiple test sessions and/or health problems, the working sample consists of 291 participants uniformly distributed across 31 age bins that covered the age range between 6 and 89 years. In light of differential rates of developmental changes across the life span, 1-, 4-, and 3-year age bins were used for age stratification for ages 6-15, 16-59, and 60-89, respectively. To study lifespan differences in the relations between stimulus-response conflict monitoring, processing fluctuation, and fluid intelligence, the sample was further divided into three continuous age groups that cover (1) childhood to adolescence (6-17 years), (2) adulthood (18-55 years), and (3) late adulthood and old age (56-89 years).

\section{Experimental task and procedure}

The task we used to assess stimulus-response conflict cost was a variant of the visual flanker task (Eriksen \& Eriksen, 1974). The task was controlled by a custom-made program running on standard PCs connected to external response keypads via serial cables. The stimuli consisted of one target circle in the center that was flanked by four other circles that were above, below, to the right, and to the left of the target. The color of the center circle was either red or green, each associated with a unique response key. The task of the participants was to press the appropriate response key on each trial depending on the color of the center circle. The task included three conditions. Within each trial, after an inter-trial interval of $1,200 \mathrm{~ms}$ a fixation cross was presented in the center of the computer screen for $500 \mathrm{~ms}$, which was followed by a blank screen for $500 \mathrm{~ms}$. The target stimulus (either a red or a green circle of about $70 \mathrm{~mm}$ in diameter) was then presented on the screen. The target circle was surrounded by four flanker circles that were $1 \mathrm{~cm}$ above, below, to the right, and to the left. In the compatible condition, the color of off-center circles (the flankers) was identical to the target circle. In the neutral condition, the color of the flankers was blue, which was neutral to either of the two response keys. In the incompatible condition, the color of the flankers not only differed from the color of the center circle and was, at the same time, identical to the color that required a competing response. Participants were instructed to respond as fast as possible to the color of the central target by pressing one of the two response keys. The task was programmed such that no response could be made either faster than $100 \mathrm{~ms}$ or longer than 2,500 ms after the onset of the stimuli. If a correct response was made, a blank screen followed for $1,200 \mathrm{~ms}$ until the next trial started again. If an incorrect response was made, an error feedback sign was given on the screen for $1,000 \mathrm{~ms}$, and then the blank screen followed for $1,200 \mathrm{~ms}$ until the next trial started.

The experiment started with 18 practice trials (three practice blocks of six trials that included randomized presentations of two stimuli for each compatibility conditions). After the experimenter made sure that the participants understood all three task conditions, the experimental session comprised seven blocks of 18 trials each, resulting in a total of 126 trials. Within each block the order of the three task conditions was randomized across trials. Given that overall error rate in this task is usually low even in young children (e.g., Rueda, Posner, \& Rothbart, 2005a) and in order to reduce age differences in speed-accuracy tradeoff when comparing response conflict score in terms of reaction time (RT) cost, the custom-made program was designed to arrive at roughly equal error rates across age groups, such that RT is the main performance measure of this task. Specifically, this was done by given additional trials (up to a maximum of 18) of corresponding trial type based on the number of incorrect response the participant made. In our data, on average the numbers of errors were minimum, even for the incongruent condition: the error rate was $3.5 \%$ for children (6-11 years old), $2 \%$ for teenagers (12-17 years old), $1.5 \%$ for younger adults (18-35 years old), $1 \%$ for middle aged adults, and $1 \%$ for older adults.

Measures of cognitive mechanics and processing fluctuation

Other than assessing individual differences in conflict monitoring, we also measured the so-called fluid intelligence (Gf), sometimes also known as cognitive mechanics, the aspect of intelligence that is presumably more directly related to information-processing and neurobiological mechanisms. For this purpose, psychometric tests used in the Berlin Aging Study (see Lindenberger \& Baltes, 1997) were adapted also for younger age groups across the lifespan (see Li et al., 2004). Data from tests reflecting basic cognitive mechanics were of particular interest here, which included three primary intellectual abilities: speed (measured by digit-letter substitution, digit-symbol substitution, identical picture tests), memory (measured by activity, 
paired associate, and text recall tests), and reasoning (measured by tests of figural analogies, letter series, and practical problems).

Furthermore, we also measured within-person processing fluctuation, given emerging evidence as reviewed above suggesting that it may reflect individual differences as well as aging-related differences in dopaminergic modulation. Specifically, processing fluctuation was assessed as a composite measure that was based on trial-by-trial RT variability in a range of elementary cognitive processing tasks, including visual search, long-term and short-term memory search, simple and choice reactions, in addition to the flanker task (see Li et al., 2004 for details about these tasks).The spread of a RT distribution generally increases linearly with the mean of the distribution (Wagenmakers \& Brown, 2007). However, an increasing body of evidence reveals that measures of trial-to-trial RT variability are indicative of individual differences in genotypes (see Castellanos \& Tannock, 2002; Winterer \& Weinberger, 2004, for reviews), in longitudinal declines of cognitive abilities during aging (e.g., Lövdén et al., 2007) and in the functional status of brain structure, neurotransmission, and neuronal activities (see a MacDonald et al., 2006, for review). Thus, current consensus is that measures of RT variability in and of themselves are important variables to be examined, in addition to mean RTs. Different approaches have been proposed to adjust for individual differences in mean RTs when analyzing processing fluctuation. Here we took the approach of regressing out trends of mean RTs across trial and control for individual differences in baseline RTs in relevant analyses. For each task, the measure of processing fluctuation was first computed by linearly regressing out trends across trials for each test conditions for each subject separately. Standard deviations of these residualized RTs were then computed for each individual and each task conditions to indicate the extent of fluctuations in RTs (cf. Williams et al. 2005). Given that the extent of fluctuations in residualized RTs may differ between tasks, standardized scores were used to compute a final composite measure of processing fluctuation that was the unit-weighted average of processing fluctuations of the various elementary cognitive tasks.

\section{Results}

U-shaped lifespan age gradient of conflict cost

To indicate age differences in the efficacy of conflict monitoring, for each participant we computed a cost score by subtracting median RT of the congruent trials from the median RT of the incongruent trials. Median RT is less sensitive to the skewness of the RT distribution than mean RT.
The longer it took the participant to react in the incongruent trial given the conflict, the greater the conflict cost indicating less efficacious conflict monitoring. The cross-sectional age gradient of conflict monitoring across the life span is shown in Fig. 1. The fitted curve was based on the group means of the 31 age bins and was derived from a combined exponential decay and increase function of conflict cost (cf. Cerella \& Hale, 1994). The fitted curve captured the Ushaped lifespan developmental function and accounted for $71 \%(r=0.84)$ of the variance in the data. Conflict cost decreases steeply from early childhood to early adulthood, remain stable in adulthood, and in early old age monitoring cost starts to gradually increase again. Conflict costs of individuals in the two youngest age bins (6-7 and 7-8 years old) and the two oldest age bins (84-86 and 87-89 years old) are, respectively, three and two times more than the cost of individuals in the age range from 25 to 50 (Fig. 1).

Relations between age differences in conflict cost, processing fluctuation, and fluid intelligence

To examine and compare lifespan age gradients of conflict cost, processing fluctuation, and cognitive mechanics $(G f)$, measures of these three variables were first transformed into the common $\mathrm{T}$ score metric. As shown in Fig. 2, lifespan age gradients of conflict cost and processing fluctuation parallel each other closely (Fig. 2, left panel). Conflict cost and processing fluctuation reduce steadily from 6 to about 20 years of age, reach a minimum

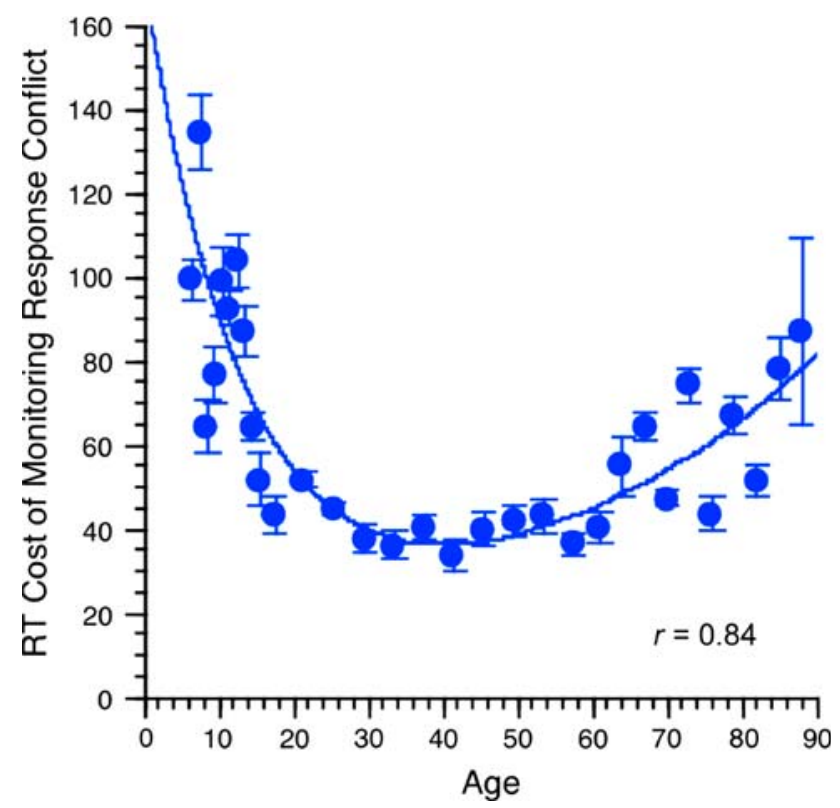

Fig. 1 Lifespan age gradient of stimulus-response conflict. Conflict monitoring score is computed as the cost of additional reaction time required for processing the flanker task in the stimulus-response incongruent condition in comparison to the time required for the congruent condition 
Fig. 2 Parallel lifespan age gradients of conflict cost, processing fluctuation (left panel), and fluid intelligence (right panel). To plot the different measures on the same scale, variables were transformed into the T-score metric
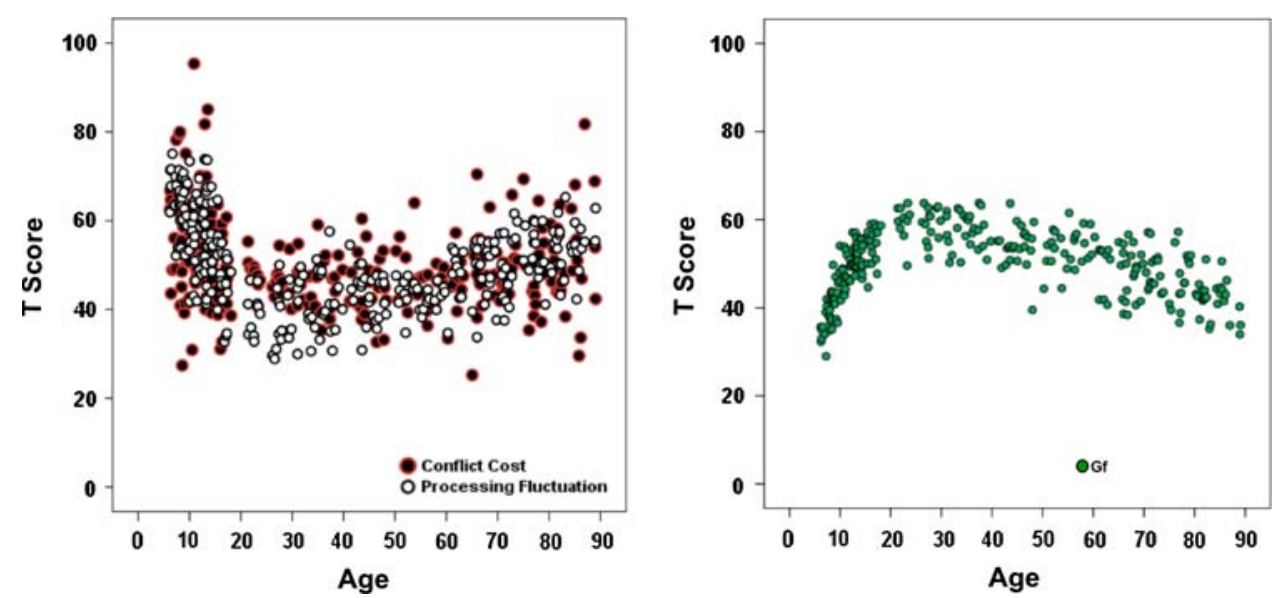

cost or fluctuation level in early 20 s, indicating that optimal efficacy in these two aspects of functioning is reached by early adulthood. The age gradients remain stable from early adulthood until about mid-50s; thereafter, conflict cost and processing fluctuation increase again. Similarly, the age gradient of fluid intelligence indicates that primary intellectual abilities in the domain of fluid intelligence $(G f)$ develop rapidly from 6 to about 20 years of age, reaches maximum in early 20 s and remains stable until early 40s, and declines thereafter. The parallel age gradients of conflict cost, processing fluctuation, and fluid intelligence suggest that they may be related to each other, either because they share some common underlying mechanisms (e.g., maturation and senescence of neurochemical and neurofunctional mechanisms) or because elementary cognitive processes, such as conflict monitoring and processing fluctuation, contribute to the development and aging of primary intellectual abilities.

To examine these relations, we separated the sample into three continuous age groups covering childhood to adolescence (6-17 years old), adulthood (18-55 years old), late adulthood and old age (56-89 years old). Two separate sets of hierarchical regression analyses were then conducted to examine the relation between conflict monitoring and processing fluctuation and between conflict monitoring and fluid intelligence.

With respect to fluid intelligence, results in Table 1 and Fig. 3 show that in terms of raw correlations, individual differences in conflict cost and fluid intelligence were significant in the child developmental and in the aging subsamples. It should be noted, however, raw correlations in this case could be confounded with individual differences in baseline RT as well as chronological age. Hierarchical regressions were thus conducted to adjust for baseline RT and age. The results show that the correlations between conflict monitoring cost and fluid intelligence are still significant and account for about 6 and $4 \%$ of individual differences in adults and older adults (Table 1, Fig. 3).
Table 1 Hierarchical regressions of conflict cost, baseline reaction time, and age on cognitive mechanics and processing fluctuation

\begin{tabular}{|c|c|c|}
\hline Age group & $\begin{array}{l}\text { Cognitive } \\
\text { mechanics }(G f)\end{array}$ & $\begin{array}{l}\text { Processing } \\
\text { fluctuation }\end{array}$ \\
\hline \multicolumn{3}{|l|}{$6-17$ years } \\
\hline Raw $R^{2}$ & $0.073 * *$ & $0.071 * *$ \\
\hline \multicolumn{3}{|c|}{ Hierarchical regression } \\
\hline Predictor & Incremental $R^{2}$ & \\
\hline Baseline RT & $0.44 * * *$ & $0.43 * * *$ \\
\hline Age & $0.30 * * *$ & $0.071 * *$ \\
\hline Conflict cost & 0.003 & 0.009 \\
\hline \multicolumn{3}{|l|}{$18-55$ years } \\
\hline Raw $R^{2}$ & 0.03 & 0.006 \\
\hline \multicolumn{3}{|c|}{ Hierarchical regression } \\
\hline Predictor & Incremental $R^{2}$ & \\
\hline Baseline RT & $0.071 *$ & $0.30 * * *$ \\
\hline Age & $0.20 * * *$ & 0.02 \\
\hline Conflict cost & $0.057 * *$ & 0.004 \\
\hline \multicolumn{3}{|l|}{$56-89$ years } \\
\hline Raw $R^{2}$ & $0.093 * *$ & $0.071 * *$ \\
\hline \multicolumn{3}{|c|}{ Hierarchical regression } \\
\hline Predictor & Incremental $R^{2}$ & \\
\hline Baseline RT & $0.12 * * *$ & $0.30 * * *$ \\
\hline Age & $0.25 * * *$ & $0.14 * * *$ \\
\hline Conflict cost & $0.04 *$ & $0.04 *$ \\
\hline
\end{tabular}

$* p<0.05, * * p<0.01, * * * p<0.001$

With respect to processing fluctuation, results in Table 1 and Fig. 4 show that in terms of raw correlations, the relations between conflict cost and processing fluctuation were significant both in the child developmental and the aging subsamples. Results from hierarchical regressions after adjusting for baseline RT and age show that conflict cost still correlated significantly with processing fluctuation (4\% of variance), but only in late adulthood and old age (Table 1, Fig. 4). 
Fig. 3 Relations between conflict cost and fluid intelligence before (left) and after (right) controlling for baseline RT and age
Fig. 4 Relations between conflict cost and processing fluctuation before (left) and after (right) controlling for baseline RT and age

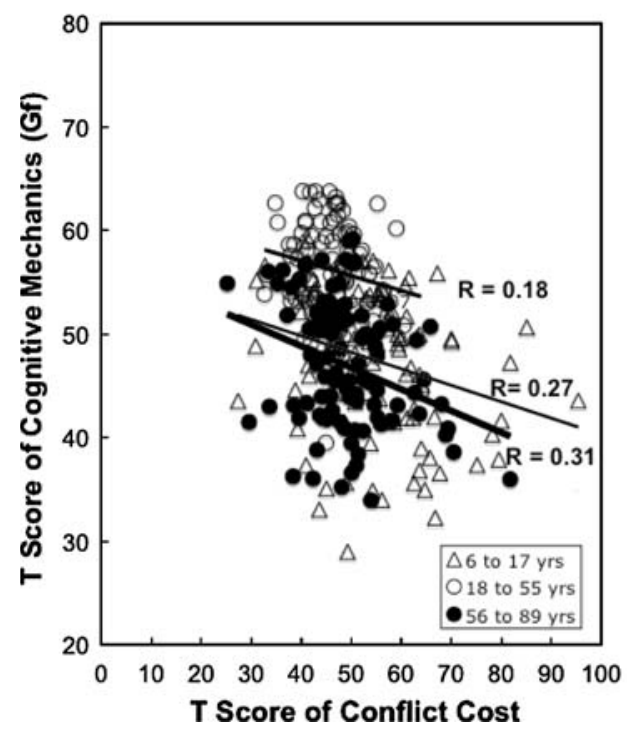

Controlling for baseline RT and Age
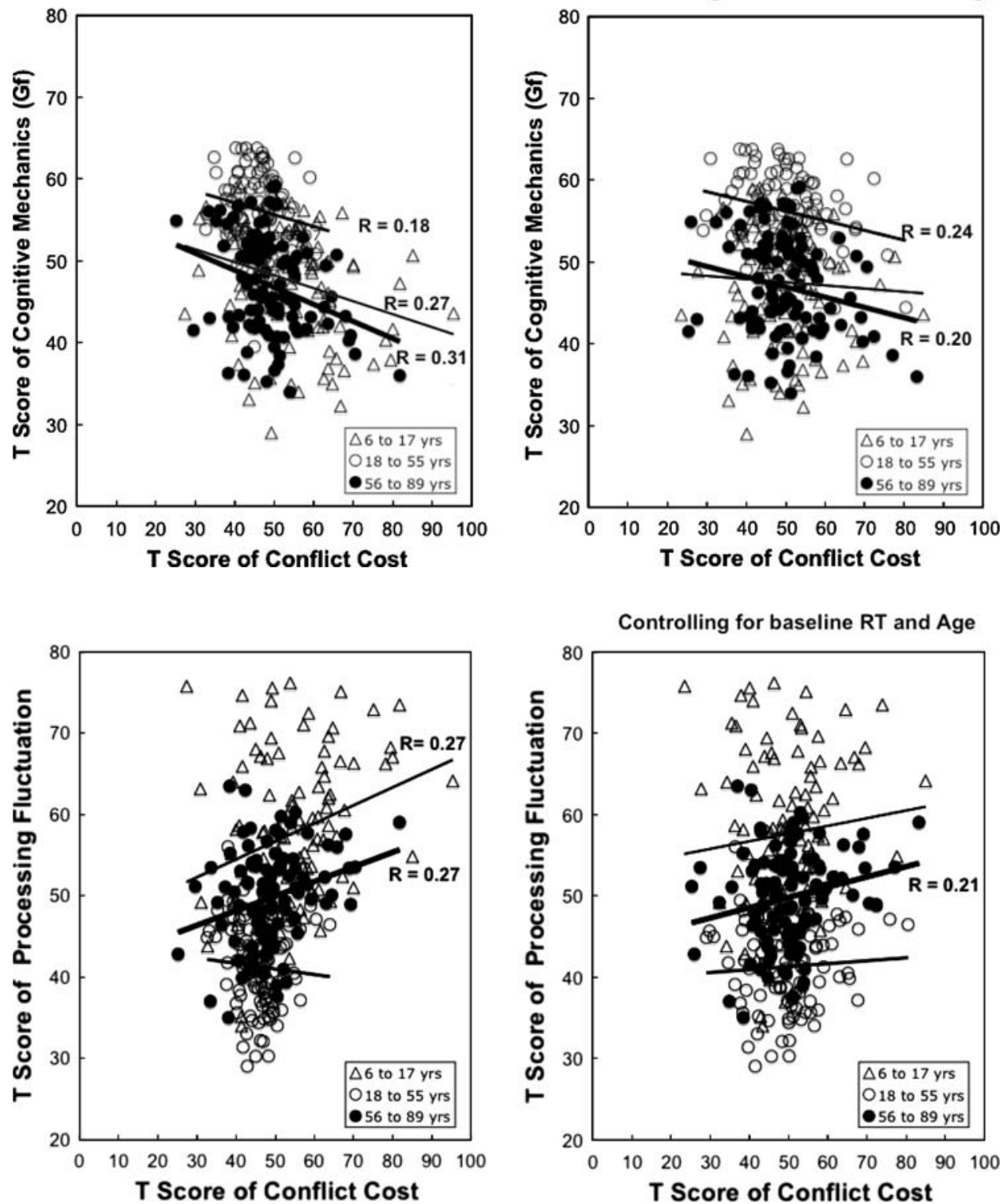

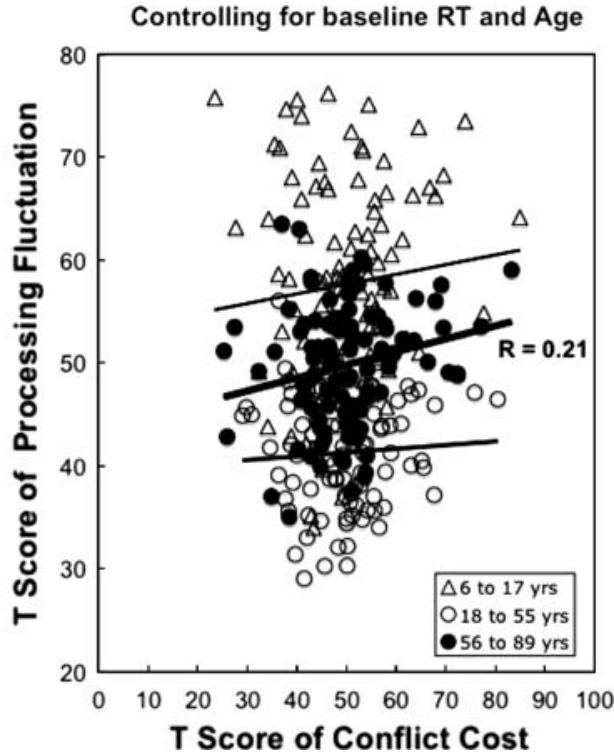

\section{Discussion}

Findings from this study reveal that, similar to other basic cognitive processes and intellectual abilities, the age gradient of conflict monitoring follows a U-function across the lifespan: with a slightly steeper growth gradient during childhood and adolescence and a more gradual declining gradient during late adulthood and old age. Furthermore, lifespan age gradient of conflict monitoring parallels very closely to the gradient of processing fluctuation at one level and the gradient of fluid intelligence at another level. Individuals at both ends of the lifespan yielded larger conflict cost and at the same time displayed a greater extent of processing fluctuations in elementary cognitive processes and a lower level of performance in tasks assessing fluid intelligence. Individuals in adulthood showed least conflict cost, least processing fluctuation, and best performance in measures of fluid intelligence.

The details of how these three aspects of functions related to each other, however, differed across the lifespan. During childhood and adolescence, the associations between conflict monitoring and process fluctuation and between conflict monitoring and fluid intelligence are primarily shared with individual differences in baseline processing speed and chronological age. After controlling for individual differences in processing speed and age, conflict monitoring no longer correlates with either processing fluctuation or cognitive mechanics. In adulthood, after controlling for individual differences in processing speed and age, conflict monitoring still accounts for a small but reliable amount of variance in fluid intelligence. In late adulthood and old age, conflict monitoring remained to be significantly 
correlated with fluid intelligence and, importantly, with processing fluctuation, even after controlling for baseline processing speed and age. The fact that, during childhood and adolescence, the contribution of conflict cost to fluid intelligence is not independent of individual differences in baseline processing speed and in other factors correlated with chronological age indicates less differentiated processes in premature cognitive systems. Thus, measures of conflict monitoring mechanisms in children may be less task specific, and reflect additional processes that affecting processing speed and other factors. More generally speaking, this finding of lifespan differences in the correlations between conflict cost and other aspects of cognition highlights the developmental dynamics in conflict monitoring mechanisms. As brain and behavioral processes undergo functional reorganizations throughout development across the lifespan (Johnson, 2001; Li et al., 2004; Lindenberger, Li, \& Bäckman, 2006; Park et al., 2004), conflict monitoring mechanisms may involve different brain functional circuitries and may affect different constellations of highlevel cognitive abilities.

The significant relation between conflict cost and fluid intelligence in adulthood and old age suggests that conflict monitoring is a putative basic cognitive mechanism that subserves intellectual abilities. In childhood, conflict monitoring also contributes to the functioning of primary intellectual abilities; however, processing speed and other factors covariate with age play more important roles. The unique association between conflict cost and processing fluctuation in late adulthood and old age lends further support to the neuromodulation of neuronal noise theory of cognitive aging (e.g., Li et al., 2001; Li, von Oertzen, \& Lindenberger, 2006) and theories on dopamine's involvement in conflict monitoring (e.g., Frank et al., 2004; Holroyd \& Coles, 2002; Yeung et al., 2004). On the one hand, empirical data and simulation results suggest that agingrelated deficit in dopaminergic modulation contributes to greater processing fluctuation, and on the other hand, dopamine is postulated to be involved in different aspects of conflict monitoring, including the more basic process, response conflict monitoring as examined here (Frank et al., 2004; see Montague et al., 2004, for review). Specifically, dopamine D1 receptor has been found to implicate the binding of perception with action (Colzato \& Hommel, 2008 , in press), which could be directly relevant to individual differences in conflict cost that arises from the incongruence between the stimulus feature and response action as examined here. Effects of dopaminergic modulation on cognitive processes have also been investigated with respect to child development (e.g., Castellanos \& Tannock, 2002; Diamond, 1996; Rueda, Rothbart, McCandliss, Saccomanno, \& Posner, 2005b). We found a relation between processing fluctuation and conflict cost in children and adolescence as well. However, this effect is mostly shared with processing speed and age, indicating that mechanisms in addition to neuromodulation of processing noise may add further influences here.

Furthermore, there is also evidence showing that individual differences in brain activity related to conflict processing are genetically predisposed (Anokhin, Heath, \& Mayers, 2004). Currently there are rapid developments in combining genomic approaches with functional brain imaging and cognitive experiments in understanding the relations between neuromodulation of functional brain processes and cognition in children and adolescence (e.g., Cornish and Hollis 2002; Rueda et al., 2005a; Wahlstrom et al., 2007), in adults (e.g., Frank, Moustafa, Haughey, Curran, \& Hutchison, 2007), during aging (e.g., Bäckman, Nyberg, \& Farde, 2006a; Nagel et al., 2008) or in the case of pathology (e.g., Frank et al., 2002; Winterer \& Weinberger, 2004). Future research on lifespan development of conflict monitoring could benefit from taking a combined genomic neurocognitive research to disentangle individual and developmental differences in gene-brain-behavior relations.

\section{References}

Anokhin, A. P., Heath, A. C., \& Mayers, E. (2004). Genetics, prefrontal cortex, and cognitive control: A twin study of event-related brain potentials in a response inhibition task. Neuroscience Letters, 368, 314-318.

Bäckman, L., Nyberg, L., \& Farde, L. (2006a). Dopamine and cognitive aging: A strong relationship. In Q. Jing, M. R. Rosenzweig, G. d'Ydewalle, H. Zhang, H.-C. Chen, \& K. Zhang (Eds.), Progress in psychological science around the world. Vol. 1: Neural, cognitive, and developmental issues (pp. 455-469). London: Psychology Press.

Bäckman, L., Nyberg, L., Lindenberger, U., Li, S.-C., \& Farde, L. (2006b). The correlative triad among aging, dopamine, and cognition: Current status and future prospects. Neuroscience Biobehavioral Reviews, 30, 791-807.

Berns, G. S., McClure, S. M., Pagnoni, G., \& Montague, P. R. (2001). Predictability modulates human brain response to reward. The Journal of Neuroscience, 27, 2793-2798.

Brown, J. W., \& Braver, T. S. (2005). Learned predictions of error likelihood in the anterior cingulated cortex. Science, 307, 1118-1121.

Botvinick, M. M., Braver, T. S., Barch, D. M., Carter, C. S., \& Cohen, J. D. (2001). Conflict monitoring and cognitive control. Psychological Review, 108, 624-652.

Botvinick, M. M., Cohen, J. D., \& Carter, C. S. (2004). Conflict monitoring and anterior cingulate cortex: An update. Trends in Cognitive Sciences, 8, 539-546.

Castellanos, F. X., \& Tannock, R. (2002). Neuroscience of attentiondeficit/hyperactivity disorder: The search for endophenotypes. Nature Reviews Neuroscience, 3, 617-628.

Cerella, J., \& Hale, S. (1994). The rise and fall in information-processing rates over the life-span. Acta Psychologica, 86, 109-197.

Cohen, J. D., Aston-Jones, G., \& Gilzenrat, M. S. (2004). A systemslevel perspective on attention and cognitive control: Guided activation, adaptive gating, conflict monitoring, and exploitation vs. exploration. In M. I. Posner (Ed.), Cognitive neuroscience of attention (pp. 71-90). New York: Guilford Press. 
Colzato, L. S., \& Hommel, B. (2008). Cannabis, cocaine, and visuomotor integration: Evidence for a role of dopamine D1 receptors in binding perception and action. Neuropsychologia, in press.

Cornish, K., \& Hollis, C. (2002). Differential impact of the dopamine transporter gene on inhibition, attentional switching and working memory functioning in children with attention deficit: A neuropsychological perspective. Archives of Clinical Neuropsychology, 17, 787-788.

Davies, P. L., Segalowitz, S. J., \& Gavin, W. J. (2004). Development of response-monitoring ERPs in 7- to -25 year-olds. Developmental Neuropsychology, 25, 355-376.

Diamond, A. (1996). Evidence for the importance of dopamine for prefrontal cortex functions early in life. Philosophical Transactions of the Royal Society of London: Biological Sciences, 351, 1483-1493.

Diamond, A., Briand, L., Fossella, J., \& Gehlbach, L. (2004). Genetic and neurochemical modulation of prefrontal cognitive functions in children. American Journal of Psychiatry, 161, 125-132.

Dolan, R. J., Fletcher, P., Frith, C. D., Friston, K. J., Frackowiak, R. S., \& Grasby, P. M. (1995). Dopaminergic modulation of impaired cognitive activation in the anterior cingulate cortex in schizophrenia. Nature, 378, 180-182.

Drewing, K., Aschersleben, G., \& Li, S.-C. (2006). Sensorimotor synchronization across the life span. International Journal of Behavioral Development, 30, 280-287.

Egner, T., \& Hirsh, J. (2005). Cognitive control mechanisms resolve conflict through cortical amplification of task-relevant information. Nature Neuroscience, 8, 1784-1790.

Eriksen, B. A., \& Eriksen, C. W. (1974). Effects of noise letters upon the identification of a target letter in a nonsearch task. Perception and Psychophysics, 16, 143-149.

Fernandez-Duque, D., \& Black, S. E. (2006). Attentional networks in normal aging and Alzheimer's disease. Neuropsychology, 20, 133-143.

Frank, M. J., Moustafa, A. A., Haughey, H. M., Curran, T., \& Hutchison, K. E. (2007). Genetic triple dissociation reveals multiple roles for dopamine in reinforcement learning. Proceedings of the National Academy of Sciences, USA, 104, 16311-16316.

Frank, M. J., Seeberger, L. C., \& O’Reilly, R. C. (2004). By carrot or by stick: Cognitive reinforcement learning in Parkinsonism. Science, 306, 1940-1943.

Higgins, J. R., \& Angel, R. W. (1970). Correction of tracking errors without sensory feedback. Journal of Experimental Psychology, $84,412-416$.

Holroyd, C., \& Coles, M. G. H. (2002). The neural basis of human error processing: Reinforcement learning, dopamine and errorrelated negativity. Psychological Review, 109, 679-709.

Hommel, B., Li, K. Z. H., \& Li, S.-C. (2004). Visual search across the lifespan. Developmental Psychology, 40, 545-558.

Johnson, M. H. (2001). Functional brain development in humans. Nature Review Neuroscience, 2, 475-483.

Ladouceur, C. D., Dahl, R. E., \& Carter, C. S. (2007). Development of action monitoring through adolescence into adulthood: ERP and source localization. Developmental Science, 10, 874-891.

Li, S.-C., Lindenberger, U., Hommel, B., Aschersleben, G., Prinz, W., \& Baltes, P. B. (2004). Transformations in the couplings among intellectual abilities and constituent cognitive processes across the lifespan. Psychological Science, 15, 155-163.

Li, S.-C., Lindenberger, U., \& Sikström, S. (2001). Aging cognition: From neuromodulation to representation. Trends in Cognitive Sciences, 5, 479-486.

Li, S.-C., von Oertzen, T., \& Lindenberger, U. (2006). A neurocomputational model of stochastic resonance and aging. Neurocomputing, 69, 1553-1560.

Lindenberger, U., \& Baltes, P. B. (1997). Intellectual functioning in old and very old age: Cross-sectional results from the Berlin Aging Study. Psychology and Aging, 12, 410-432.
Lindenberger, U., Li, S.-C., \& Bäckman, L. (2006). (Eds.). Brainbehavior dynamics across the lifespan. Neuroscience and Biobehavioral Reviews, 30, 713-885.

Lövdén, M., Li, S.-C., Shing, Y. L., \& Lindenberger, U. (2007). Within-person trial-to-trial variability precedes and predicts cognitive decline in old and very old age: Longitudinal data from the Berlin Aging Study. Neuropsychologica, 45, 2827-2838.

MacDonald, S. W. S., Hultsch, D. F., \& Dixon, R. A. (2003). Performance variability is related to change in cognition: Evidence from the Victoria Longitudinal Study. Psychological Aging, 18, 510 523.

MacDonald, S. W. S., Nyberg, L., \& Bäckman, L. (2006). Intraindividual variability in behavior: Links to brain structure, neurotransmission and neuronal activity. Trends in Neuroscience, 29 , 474-480.

MacDonald, S. W. S., Nyberg, L., Sandblom, J., Fischer, H., \& Bäckman, L. (2008). Increased response-time variability is associated with reduced inferior parietal activation during episodic recognition in aging. Journal of Cognitive Neuroscience, 20, 779-786.

Marschner, A., Mell, T., Wartenburger, I., Villringer, A., Reischies, F. M., \& Heekeren, H. R. (2005). Reward-based decision-making and aging. Brain Research Bulletin, 67, 382-390.

Mathalon, D. H., Bennett, A., Askari, N., Gray, E. M., Rosenbloom, M. J., \& Ford, J. M. (2003). Response-monitoring dysfunction in aging and Alzheimer's disease: An event-related potential study. Neurobiology of Aging, 24, 675-685.

Mathewson, K. J., Dywan, J., \& Segalowitz, S. J. (2005). Brain bases of error-related ERPs as influenced by age and task. Biological Psychology, 70, 88-104.

Montague, P. R., Hyman, S. E., \& Cohen, J. D. (2004). Computational roles for dopamine in behavioral control. Nature, 431, 760-767.

Nagel, I. E., Chicherio, C., Li, S.-C., Oertzen, V., Sander, T., Villringer, A., et al. (2008). Human aging magnifies genetic effects on executive functioning and working memory. Frontiers in Human Neuroscience, 2, 1-8.

Nieuwenhuis, S., Ridderinkhof, K. R., Talsma, D., Coles, M. G. H., Holroyd, C. B., Kok, A., et al. (2002). A computational account of altered error processing in older age: Dopamine and error-related processing. Cognitive, Affective, and Behavioral Neuroscience, 2, 19-36.

Park, D. C., Polk, T. A., Park, R., Minear, M., Savage, A., \& Smith, M. R. (2004). Aging reduces neural specialization in ventral visual cortex. Proceedings of the National Academy of Science, USA, 101, 13091-13095.

Rabbitt, P. M. A. (1966). Errors and error correction in choice response tasks. Journal of Experimental Psychology, 71, 264-272.

Raz, N., Lindenberger, U., Rodrigue, K. M., Kennedy, K. M., Head, D., Williamson, A., et al. (2005). Regional brain changes in aging healthy adults: General trends, individual differences, and modifiers. Cerebral Cortex, 15, 1676-1689.

Ridderinkhof, K. R., Ullsperger, M., Crone, E. A., \& Nieuwenhuis, S. (2004). The role of the medial frontal cortex in cognitive control. Science, 306, 443-447.

Ridderinkhof, K. R., \& van den Wildenberg, W. P. (2005). Neuroscience. Adaptive coding. Science, 307, 1059-1060.

Rueda, M. R., Fan, J., McCandliss, B. D., Halparin, J. D., Gruber, D. B., \& Lercari, L. P. (2004). Development of attentional networks in childhood. Neuropsychologia, 42, 1029-1040.

Rueda, M. R., Posner, M. I., \& Rothbart, M. K. (2005a). The development of executive attention: Contributions to the emergence of self-regulation. Developmental Neuropsychology, 28, 573 594.

Rueda, M. R., Rothbart, M. K., McCandliss, B. D., Saccomanno, L., \& Posner, M. I. (2005b). Training, maturation, and genetic influences on the development of executive attention. Proceedings of the National Academy of Sciences, USA, 102, 14931-14936. 
Santesso, D. L., Segalowitz, S. J., \& Schmidt, L. A. (2006). Error-related electrocortical responses in 10-year-old children and young adults. Developmental Science, 5, 473-481.

Schott, B. H., Sellner, D. B., Lauer, C. J., Habib, R., Frey, J. U., Guderian, S., et al. (2004). Activation of midbrain structures by associative novelty and the formation of explicit memory in humans. Learning and Memory, 11, 383-387.

Schultz, W. (2002). Getting formal with dopamine and reward. Neuron, 36, 241-263.

Sowell, E. R., Thompson, P. M., Holmes, C. J., Jernigan, T. L., \& Toga, A. W. (2003). Mapping cortical changes across the life span. Nature Neuroscience, 2, 859-861.

Ullsperger, M., \& von Cramon, D. Y. (2004). Decision making, performance and outcome monitoring in frontal cortical areas. Nature Neuroscience, 7, 1173-1174.

Wahlstrom, D., White, T., Hooper, C. J., Vrshek-Schallhorn, S., Oetting, W. S., Brott, M. J., et al. (2007). Variations in the catechol Omethyltransferase polymorphism and prefrontally guided behaviors in adolescents. Biological Psychiatry, 61, 626-632.

Waszak, F., Schneider, W. X., Li, S.-C., \& Hommel, B. (2008). Perceptual identification across the life span: A dissociation of early gains and late losses. Psychological Research, in press.
West, R. (2004). The effects of aging on controlled attention and conflict processing in the Stroop task. Journal of Cognitive Neuroscience, 16, 103-113.

West, R., \& Moore, K. (2005). Adjustments of cognitive control in younger and older adults. Cortex, 41, 570-581.

Williams, B. R., Hultsch, D. F., Strauss, E. H., Hunter, M. A., \& Tannock, R. (2005). Inconsistency in RT across the lifespan. Neuropsychology, 19, 88-96.

Williams, B. R., Ponesse, J. S., Schachar, R. J., Logan, G. D., \& Tannock, R. (1999). Development of inhibitory control across the life span. Developmental Psychology, 25, 205-213.

Winterer, G., \& Weinberger, D. R. (2004). Genes, dopamine and cortical signal-to-noise ratio in schizophrenia. Trends in Neuroscience, 27, 683-690.

Wagenmakers, E.-J., \& Brown, S. (2007). On the linear relation between standard the mean and deviation of a reaction time distribution. Psychological Review, 114, 830-841.

Yeung, N., Botvinick, M. M., \& Cohen, J. D. (2004). The neural basis of error detection: Conflict monitoring and the error-related negativity. Psychological Review, 111, 931-959. 